\title{
THE REDSHIFT DEPENDENCE OF GALAXY SURFACE BRIGHTNESS
}

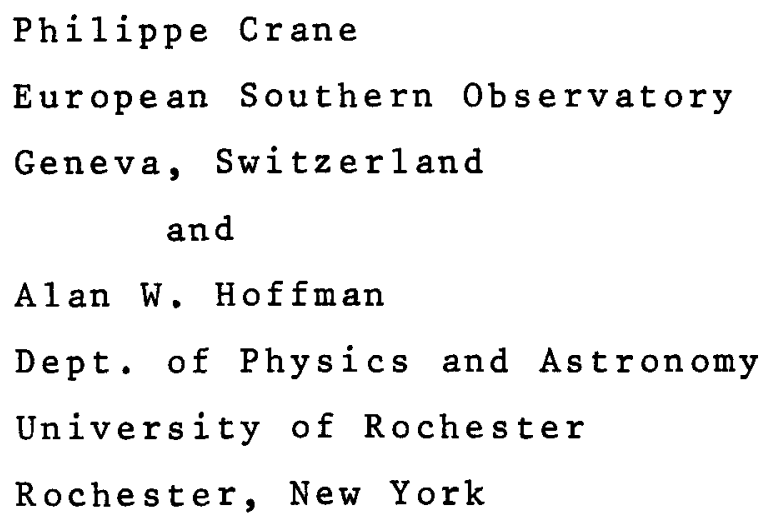

Nous avons déterminé la brillance superficielle moyenne d'amas à l'intérieur d'un diamètre linéaire projeté de $20.3 \mathrm{kpc}$ en faisant la photométrie de galaxies dans six amas ayant un redshift de $z=0.05$ à 0.20 . Pour toutes les cosmologies dans lesquelles le décalage vers le rouge est uniquement dû à l'expansion, la brillance superficielle devrait avoir la forme $m(S B)=2.5$ a $\log (1+z)+b$ où $a \equiv 4$ et $b$ est une constante d'échelle. Nous trouvons $a=4.1 \pm 1.6$ et $b=22.12 \pm 0.21$. Ces résultats supportent fortement l'interprétation des décalages vers le rouge comme dus à l'expansion de l'univers.

A cosmological model independent connection between the expansion of the Universe and observed redshifts (z) can be demonstrated if the $(1+z)^{-4}$ dependence of the surface brightness (SB) of some class of standard objects is established. The functional form $S B(z)=S_{0} /(1+z)^{4}$ follows from the Liouville theorem in a 4-dimensional expanding space-time (Misner, Thorne, and Wheeler, 1973) 
and is, hence, based on some of our most fundamental physical concepts. Apart from evolutionary effects and the like, significant departures from this predicted form would have major consequences for our understanding of the cosmos in terms of locally derived physics.

The major problem with any attempt to measure this surface brightness is to find standard objects properly distributed in space. Ideally one would like to use a set of galaxies for which an intrinsic physical length can be determined from the galaxies themselves. The luminosity within this physical length can then be used to determine an average SB. However, even this ideal procedure requires the observation of many galaxies at each redshift since there exist no true standard galaxies (Crane, 1976). Ground based attempts to follow this procedure will most certainly be frustrated by the fact that the best intrinsic physical length, the galaxy core radius (Sandage, 1974), will be much smaller than typical seeing discs at the required redshifts $(z>0.1)$.

There is another approach open to the ground based astronomer and that is to choose a physical length a priori and to project it out to the required redshifts according to some cosmological mode1. This approach unfortunately does not yield cosmologically model independent results. However, the dependence on cosmological models is relatively small at redshifts less than $z=0.3$. This is the approach which we have taken in this work.

The procedure is rather straightforward. We observed the luminosity ( $\mathrm{L}_{\mathrm{ob}}$ ) within an angular radius $\theta_{o b}(z)$. These luminosities were determined with a fixed bandwidth and 
and wavelength on the Earth and were corrected for K-dimming and galactic absorbtion. The details of these corrections can be found in Hoffman and Crane (1977 a). The quantity we wish to determine is the average surface brightness within a standard metric angular radius, $\theta_{m}(q, z)$. We have chosen a physical radius of $10.15 \mathrm{kpc}$ and used the standard Friedman cosmologies with $q_{0}=\frac{1}{2}$ (see Weinberg, 1972) and $H_{0}=50 \mathrm{Km} \mathrm{s}-1$ $\mathrm{Mpc}^{-1}$ to determine $\theta_{\mathrm{m}}\left(\frac{1}{2}, z\right)$ at the required redshifts. We must also correct $L_{o b}$ for the fact the $\theta_{o b}(z)$ might not equal $\theta_{m}(q, z)$. Following Gunn and Oke (1975), we assume that the galaxian growth curve is a power 1 aw (with power $\alpha \cong 0.75$ ) and correct $L_{o b}$ to $L_{m}=L_{o b}\left(\theta_{m}\left(\frac{1}{2}, z\right) / \theta_{o b}(z)^{\alpha}\right.$. We then determine

$$
\operatorname{SB}\left(q_{0}=\frac{1}{2}, z\right)=L_{m} / \pi \theta_{m}^{2}\left(\frac{1}{2}, z\right)
$$

If, in fact, $q_{0}=\frac{1}{2}$, then this surface brightness is the quantity to be tested for $(1+z)^{-4}$ dependence. To remove the $q_{0}$ dependence, we rewrite Equation 1

$$
S B(z)=\left\{\frac{L_{o b}}{\pi \theta_{o b}^{\alpha}(z) \theta_{m}^{2-\alpha}\left(\frac{1}{2}, z\right)}\right\}\left[\frac{\theta_{m}\left(\frac{1}{2}, z\right)}{\theta_{m}(q, z)}\right]^{2-\alpha}
$$

This is the cosmological model independent quantity which is to be used to test the $(1+z)^{-4}$ dependence of surface brightness. It should be noted that this expression contains exactly the same dependence on (or independence of) $q_{0}$ as the conventional expression for the redshift-magnitude relation. (Gunn and Oke, 1975).

According to the conventional wisdom, one should include a chemical evolution term (Larson and Tinsley, 1974) and a dynamical evolution term (Ostriker and Tremaine, 1975) on the 
right of Equation 2 if $S B(z)$ is to maintain the $(1+z)^{-4}$

dependence. Since dynamical evolution is not well understood, we will ignore it, and approximate the chemical evolution correction by (see Tinsley, 1972)

$$
\operatorname{EV}(z)=(1+z)^{-0.33}
$$

In order to illustrate the magnitude of these effects, we rewrite Equation 2

$$
\operatorname{SB}(z)=S_{O b}(z) Q(z) E V(z)
$$

where $S_{\circ b}(z)$ is the term in curly brackets in Equation 1 , and $Q(z)=\theta_{m}\left(z, \frac{1}{2}\right) / \theta_{m}(z, q) 2-\alpha$. $E V(z)$ and $Q(z)$ for $q_{0}=1.0$ and 0.06 are plotted in Figure 1 . The evolutionary term is always $1 / 12$ th of the expansion and has the effect of making the observed $q_{0}$ larger than the true $q_{0}$. The cosmological term $Q$ is also on the order of $1 / 12$ the expansion for $0.06<q_{0}<1.0$. Of course, in practice $q_{0}$ and evolution are not well determined, and cannot be applied as corrections to observation such as k-dimming is, but must be determined from the data.

In our observations, the Iuminosity, $L_{\text {ob }}$ mentioned above was, in fact, the "characteristic luminosity" L* derived from a luminosity function of the type discussed by Schechter (1976). The data were obtained from observations by Hoffman and Crane (1977 a) and include luminosities for 1086 galaxies in six clusters with redshifts ranging from $z=0.05$ to $z=0.20$. The procedures for obtaining the characteristic luminosity from these observations are available (Hoffman, 1975; Hoffman and Crane, 1977b). The table below list the data relevant to this discussion. 


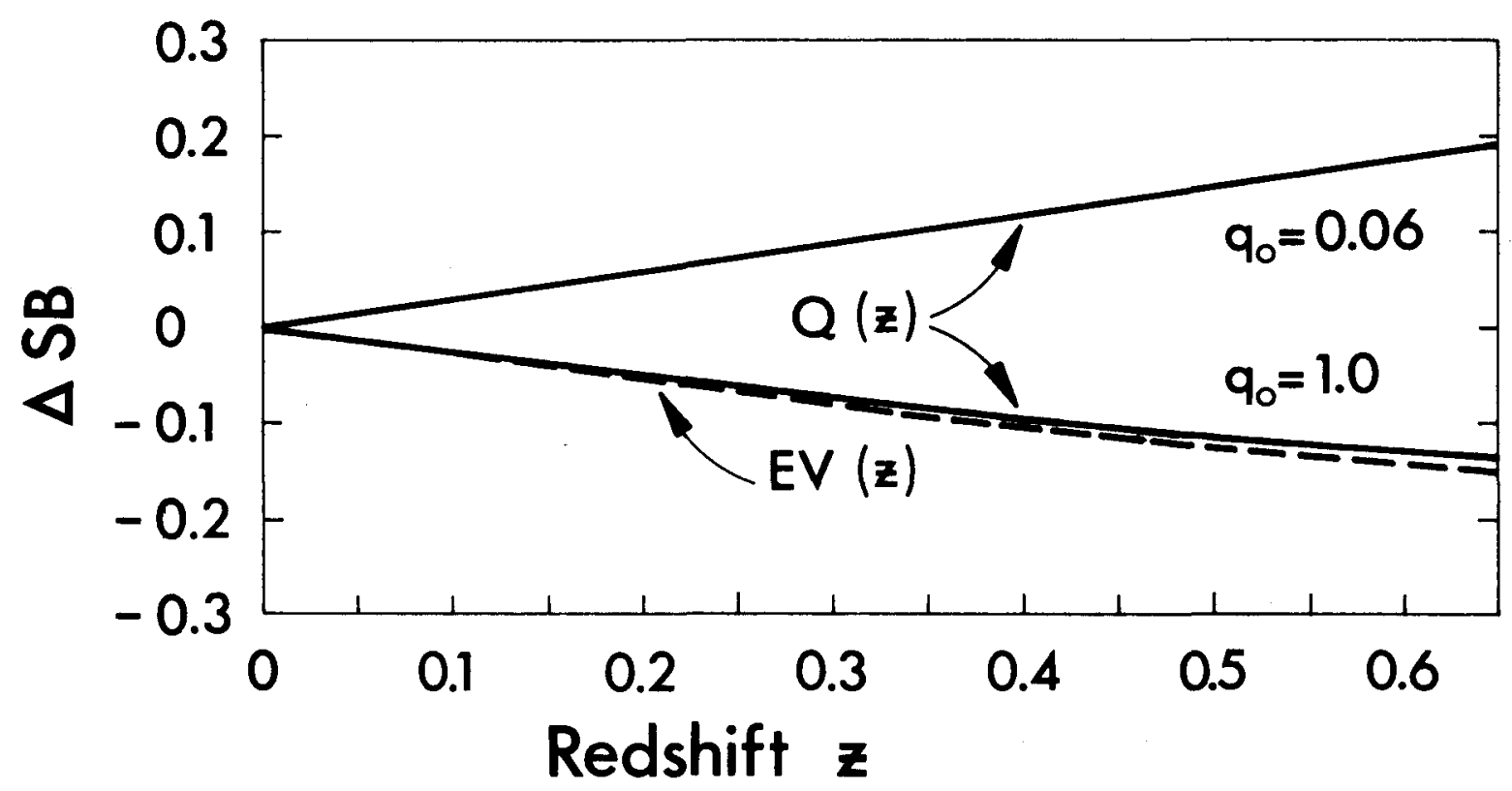

Figure 1: Corrections for evolution, $E V(z)$, and for $q_{0} \neq \frac{1}{2}$ $Q(z)$. These are corrections to be applied to the observations $S_{o b}(z)$ to remove these two effects from the data. $Q(z)$ is calculated with $\alpha=0.75$.

Table 1: Cluster Surface Brightness Data

\begin{tabular}{|c|c|c|c|c|}
\hline $\begin{array}{c}\text { Abe11 } \\
(1)\end{array}$ & $\begin{array}{l}\text { Name } \\
(2)\end{array}$ & $\begin{array}{c}z \\
(3)\end{array}$ & $\begin{array}{c}\text { (SB ob) } \\
(4)\end{array}$ & $\begin{array}{c}\sigma \\
(5)\end{array}$ \\
\hline 1377 & $\begin{array}{l}\text { Ursa } \\
\text { Major 1 }\end{array}$ & 0.0516 & 22.20 & 0.21 \\
2065 & $\begin{array}{l}\text { Corona } \\
\text { Borea1is }\end{array}$ & 0.0722 & 22.55 & 0.15 \\
1132 & $\begin{array}{l}\text { Ursa } \\
\text { Major II }\end{array}$ & 0.1345 & 22.83 & 0.16 \\
801 & - & 0.1426 & 22.58 & 0.11 \\
732 & Hydra II & 0.2018 & 23.20 & 0.30 \\
\hline
\end{tabular}

Notes to Table 1: Column 1, Abell Cluster Number (Abe11 1958); Column 2, Cluster name if any; Column 3 , Redshift in units of $\mathrm{v} / \mathrm{c}$; Column 4, Characteristic surface brightness in V magnitudes/ $(\operatorname{arcsec})^{2}, m(S B)=-2.5 \log \mathrm{SB}$; Column 5 , Uncertainty in $\mathrm{m}\left(\mathrm{SB}_{\mathrm{ob}}\right)$ same units. 
This data set has several advantages over other available data sets for the purpose of the surface brightness test. All galaxies in all clusters have been treated identical1y. Each $L_{\text {ob }}$ contains information averaged over more than a hundred galaxies per cluster and is therefore quite independent of variations in individual galaxies. We have used somewhat smaller apertures, $\theta_{\text {ob }}$, than previous observers and this tends to increase the value of $\alpha$ mentioned above and, hence, reduce the sensitivity of our results to our choice of cosmology. The individual galaxy luminosities were individually corrected for seeing effects so that inspite of our smaller apertures, we do not degrade the results because of seeing. We have eliminated the aperture correction by choosing $\theta_{o b}(z) \equiv \theta_{\text {met }}(z)$.

The data of Table 1 have been fit to a function of the form $m(S B)=2.5$ a $\log (1+z)+b$. The results are $a=4.10 \pm$ $1.62, b=22.12 \pm 0.21$ with a $x^{2}$ of 4.53 for 4 degrees of freedom. Figure 2 shows a plot of the data and the fitted result. The conventional interpretation of redshifts predicts $a \equiv 4$ in the absence of evolution. Tired light cosmologies (Geller and Peebles, 1972) or any other model in which the redshift depends only on the light travel distance (Pecker et a1., 1973) predict a $\equiv 1$. Our results rule out models with $a=1$ at the $94 \%$ confidence level. However, this must be slight1y qualified since tired light cosmologies allow varied predictions for the metric radius versus redshift relation (see Geller and Peebles 1972) and in the absence of other compeliing information, we have chosen tired light metric radii which match our $q_{0}=\frac{1}{2}$ radii at $z<0.2$. This represents a tired light cosmology in a closed curved space. For spaces with larger radii of curvature, our result is not as strong. 


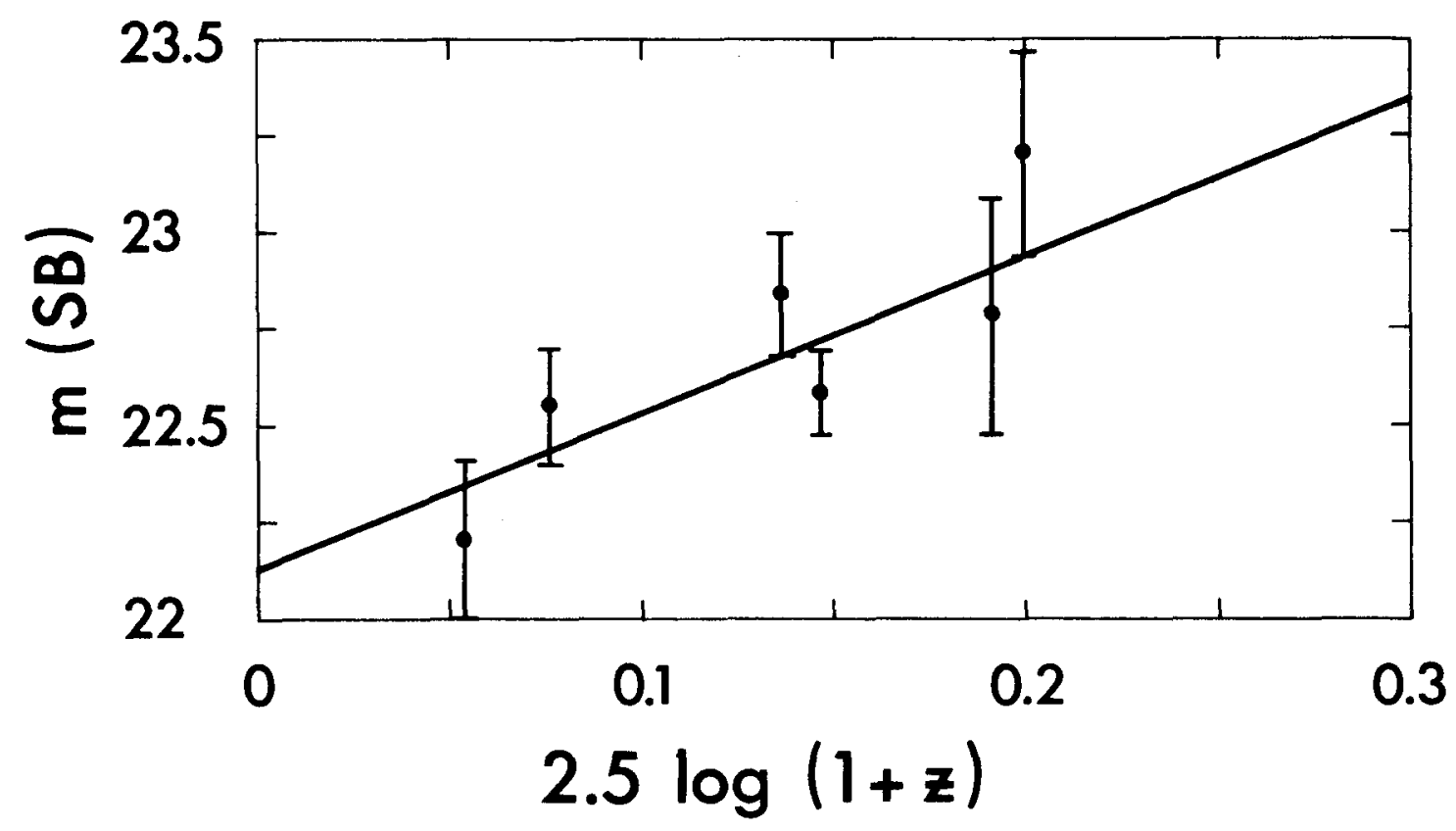

Figure 2: Plot of the observed surface brightness $\mathrm{SB}(z)$ vs $2.5 \log (1+z)$. The 1 ine has been determined from the values given in the text for our results. The units of surface brightness are magnitudes per (arcsecond) ${ }^{2}$.

In addition, we can show that the characteristic luminosity, $L^{*}$, is a good standard candle out to $z=0.20$ having a dispersion $\sigma\left(m_{v}^{*}\right)=0.19$ compared to $\sigma\left(M_{1}\right)=0.51$ for these same clusters. By design, the results contain no information on the value of 90 or on evolution. 
Crane, P., 1976,Ap.J. (Letters),206, L 133 .

Geller, M.J. and Peebles P.J.E., 1972 Ap. J., 174, 1.

Gunn, J.E., and Oke, J.B. 1975 Ap.J., 195, 255.

Hoffman, A.W. 1975, PhD. Thesis, Princeton University (unpublished)

Hoffman, A.W., and Crane P. $1977 \mathrm{a}$, Ap.J. (in press)

Hoffman, A.W., and Crane P. $1977 \mathrm{~b}$, (in preparation)

Larson, R.B., and Tinsley B.M., 1974, Ap.J., 192, 293.

Misner, C.W., Thorne, K.S., and Wheeler, J.A. 1973,

Gravitation (San Francisco: W. H. Freeman \& Co) pp 584.

Ostriker, J.P., and Tremaine, S.D., 1975, Ap.J. (Letters), 202, L 113 .

Pecker, J.C., Tait, W., and Vigier, J.P. 1973, Nature, 241, 338 .

Sandage, A.R. 1974, in Large Space Telescope, AIAA $12 \mathrm{th}$

Aero Sci. Meeting Washington D.C.

Schechter, P. 1976, Ap.J., 203, 297.

Tinsley, B.M., 1972, Ap.J. (Letters), 173, L 93.

Weinberg, S., 1972, Gravitation and Cosmology (New York:

John Wiley and Sons). 
DISCUSSION

K. RUDNICKI: How would your results be affected by the systematic observational errors connected with differences of angular diameters of galaxies with different $z$.

P. CRANE: We have chosen a specific cosmological model to determine the metric angular diameters at different redshifts. If our cosmological model is wrong, then we must modify the luminosity we measure by the amounts shown in Figure 1 . This will then affect the results accordingly, but at redshifts less than 0.2 this will be quite small.

G. DE VAUCOULEURS: 1) Did you measure a nearby cluster, such as Virgo, where the measurements could be compared with other data?

2) How many objects did you measure in each cluster and how were they calibrated?

P. CRANE: 1) For two of the clusters we have comparisons with other observers. These comparisons indicate that there are no systematic errors in the magnitudes we have determined which would affect our results.

2) We measured a minimum of about 60 and a maximum of about 300 objects in these clusters. The magnitude scales were determined by comparison with several photoelectric magnitudes in each cluster. The dispersion of our results around the photoelectric results is $\sigma \approx 0.05$ magnitudes. V. PETROSIAN: The main difference between the tired-light and expanding models - the two powers of $(I+z)$ - come from the absence of abberation in the tired-light model. This must be taken into account in the correction term $Q(z)$. Once this is done in the present analysis the expected relation between surface brightness and redshift in the tired-light model will be approximately $\mathrm{SB}(\mathrm{z}) \sim 1+\mathrm{z})^{-3+\alpha}$, so that the tired-light model can be ruled out only at one $\sigma$ level. I would like to emphasize that the insensitivity of the surface brightness test is basic (as described by myself and B. Tinsley; cf. figure 1 of my paper on surface brightness) and cannot be overcome by a different analysis. These comment apply also to Spinrad's results. 